\title{
Primary Healthcare Nursing Cadre in India: Needs Paradigm Shift
}

\author{
Divya Aggarwal, Sumant Swain and Sanjiv Kumar \\ International Institute of Health Management Research, New Delhi, India \\ *Corresponding author: Sanjiv Kumar, International Institute of Health Management Research, New Delhi, India
}

\section{Introduction}

Nursing care is the backbone of health system. Nurses role are very critical in delivering health services and increasing equity of healthcare provision. They are the agents of change within health system and play a key role in addressing various social determinants of heath. The role of nurses in primary healthcare encompasses autonomous and collaborative care of individuals of all ages, families, groups and communities, sick or well in community settings. It includes the promotion of health, the prevention of illness, and the care of ill, disabled and dying people. India is undergoing a major epidemiological transition. Over the last 26 years, the country's disease patterns have shifted. Mortality due to communicable, maternal, neonatal, and nutritional diseases (CMNNDs) has declined substantially. Non-communicable diseases (NCDs) and injuries are increasingly contributing to overall disease burden. The proportion of burden of diseases in India due to CMNNDs reduced from $53.6 \%$ in 1990 to $27.5 \%$ in 2016. NCDs related burden of diseases increased from $37.9 \%$ to $61.8 \%$ and burden due to injuries changed from $8.5 \%$ to $10.7 \%$. Life expectancy at birth improved in India from 59.7 years in 1990 to 70.3 years in 2016 for females, and from 58.3 years to 66.9 years for males. Therefore, the role of nurses in primary healthcare has changed due to the change in disease burden from CMNNDs to NCDs and injuries. Along with that they have the crucial role in recently announced Health and Wellness Centers and National Health Protection Scheme with will cover more than half a billion population in India.

\section{Current Scenario}

In India, different leadership positions are designated separately for community (Public Health) and clinical nurses at the primary health care level. Auxiliary Nurse Midwives (ANM) is the first level community nurse and its first supervisor is Lady Health
Visitor (LHV). ANMs have a crucial role in healthcare delivery as they are responsible for implementation of health programmers and extending the outreach clinical services at the community level. They coordinate with community level health volunteers called Accredited Social Health Activist (ASHAs) which are about a million in numbers and the volunteers of Women and Child Health called Anganwadi workers (AWW) for smooth delivery of healthcare services. Both ASHA and AWWs look after about one thousand population each [1-5]. The clinical nurses are categorized into Registered Nurses (RN) and Registered Midwives (RM). According to National Health Profile 2018, there are 1,980,539 RNs \& RMs, 841,279 ANMs and 56,367 LHVs serving in India as of 2016. As of 31st March 2017, the country had a short fall of 10,112 of ANMs (also called female health workers) at the Sub-center and primary health centers (PHCs), 11,712 LHVs (also called female health assistants), 15,592 male supervisors (also called male health assistants). 3,027 medical officers and 13,194 nurses at PHCs and CHCs (Community Health Centers) level. Even out of the sanctioned posts, a significant percentage of posts were vacant at the primary level. For instance, 13.59 percent ANM, 37.45 percent Health Worker (male), 34.72 percent LHV, 47.16 percent Health Assistants (Male), and 1.65 percent Nursing staff at PHCs and CHCs are vacant. India is far away from its aim of two nurses and one ANM per doctor to be achieved by the year 2025 [6,7].

\section{Role in Health \& Wellness Centres}

It has been observed that optimum utilization of Sub-Centres in meeting out their key objective is hindered due to factors like poor infrastructure, weak monitoring and supportive supervision, lack of manpower, and geographical barriers. The sub-centres do not provide services for NCDs, injuries, old age problems and mental health etc. It forces the rural community to either opt for 
treatment with quacks (unregistered medical practitioners) or travel to hospitals/ private healthcare facilities in cities which leads to unaffordable out -of- pocket expenditures. In order to improve the health seeking behavior at community, National Health Policy 2017 had envisioned to convert existing sub centres (SCs) into Health and Wellness Centres (H\& WCs) which aims at providing comprehensive primary health care delivery. This policy has also focused on developing cadres like Nurse Practitioners for better availability of basic services in primary healthcare facilities. This policy has given flexibility to states to train graduate nurses or Ayurvedic doctors for six months and to make them team leaders at the 1,25,000 upgraded sub center now called Health Wellness Centres to provide the comprehensive primary health care. At the health and wellness centres, this additional cadre of workforce also referred to as Mid-level Healthcare Providers (MLHP) will provide care for non-communicable diseases, child health services, mental health, geriatric health, essential drugs and basic diagnostic services [8-12]. The role of ANMs will expand to include providing promotive and preventive health care, first aid, basic primary healthcare (under the supervision of the MLHP) and ensure referral to higher levels of care, as appropriate. The key responsibilities of ANM can be broadly classified into six major areas: Out-patient Services, Immunization Sessions/Village Health and Nutrition Day, visits to the community, In-patient midwifery services, maintaining records \& reports, planning work, capacity building, referral of highrisk pregnancies, sick neonates and other emergencies, screening for chronic non communicable diseases and the provision of basic laboratory tests for pregnant women. Further, recently launched Ayushman Bharat scheme gives an opportunity to develop a cadre of nurse practitioners for effective implementation of National Health Protection Scheme (NHPS).

\section{Conclusion}

Due to changing pattern of demography, epidemiology of disease conditions and an augmentation of Nursing cadre role at primary healthcare institutions, it is essential to enhance capacity of nursing staff at community level. Their curriculum and job description needs to be revised to address current and emerging health challenges in India. Most of the ANMs join and retire as ANMs after 30-35 years of service in the healthcare. LHV and PHN positions to which they can be promoted to remain vacant which further demotivates them. Nurses in India prefer working in the public sector because it pays better than private sector. More than half of the nurses in India are employed by central and state governments, and opportunities for public sector employment have increased since the launch of the National Rural Health Mission in 2005. In addition, in-service training of nurses to move from ANM to LHV or PHN level through courses similar to post certificate nursing and to Nurse practitioner to lead Health \& Wellness centres should be carried out on priority. They also need management and basic leadership training to effectively manage their work and improve health outcomes.

\section{References}

1. (2018) Nursing Definitions.

2. Indian Council of Medical Research, Public Health Foundation of India, and Institute for Health Metrics and Evaluation (2017) India: Health of the Nation's States - The India State-Level Disease Burden Initiative. New Delhi, India.

3. National Health Profile (NHP) of India (2018) Central Bureau of Health Intelligence, Directorate General of Health Services, Ministry of Health and Family Welfare, Government of India.

4. Rural Health Statistics (2017) Ministry of Health and Family Welfare, Government of India.

5. High Level Expert Group Report on Universal Health Coverage for India Report, pp. 153.

6. Sanjiv Kumar, Rinu PK (2018) Leadership Skills for Nurses. JOJ Nurse Health Care 8(5): 555747.

7. National Health Policy (2017) Ministry of Health and Family Welfare, Government of India.

8. Biju BL (2013) India: Angels are turning read: Nurses' strikes in Kerala. Economic and Political Weekly 48: 52.

9. Nair S (2010) Nurses' strikes in Delhi: a status question. Economic and Political Weekly 45(14).

10. Nair KS (2015) Human resources for health in India: an overview. International Journal of Health Sciences and Research, pp. 465.

11. Reynolds J, Wisaijohn T, Pudpong N, Watthayu N, Dalliston A, et al. (2013) A literature review: the role of the private sector in the production of nurses in India, Kenya, South Africa and Thailand. Human Resources for Health 11: 14.

12. Rao KD, Bhatnagar A, Berman P (2009) India's health workforce: size, composition, and distribution. India Health Beat, Public Health Found India and World Bank, New Delhi, India 1: 3.

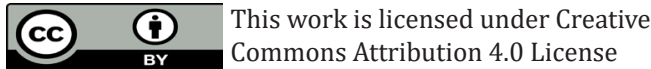

To Submit Your Article Click Here:

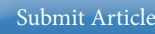

DOI: $10.32474 /$ LOJNHC.2019.02.000131

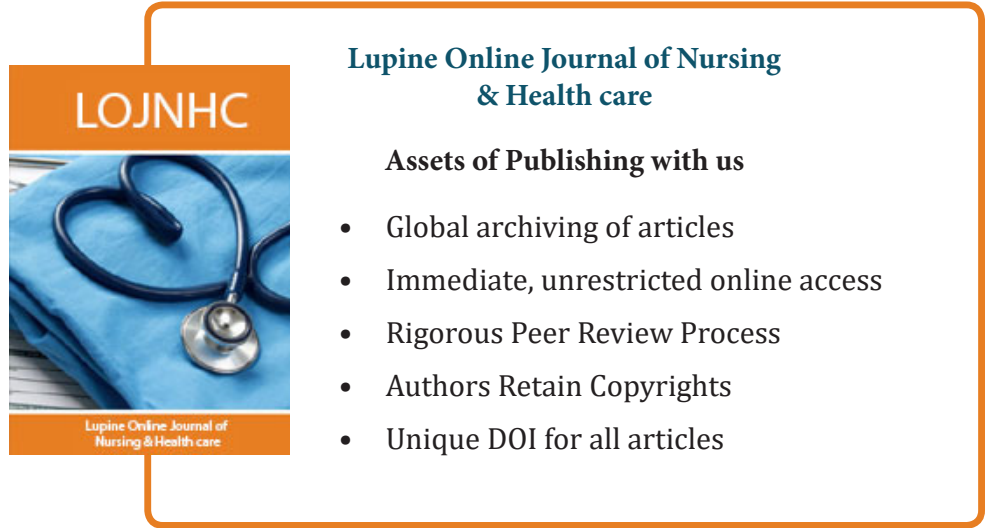

